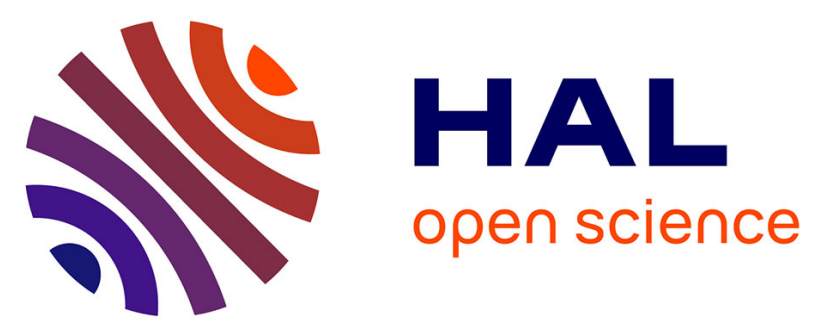

\title{
Semi-Markov processes with semi-regenerative states for the availability analysis of chemical process plants with storage units
}

\author{
Olga Fink, Enrico Zio
}

\section{- To cite this version:}

Olga Fink, Enrico Zio. Semi-Markov processes with semi-regenerative states for the availability analysis of chemical process plants with storage units. Proceedings of the Institution of Mechanical Engineers, Part O: Journal of Risk and Reliability, 2013, 227, pp.279-289. 10.1177/1748006X13480765 . hal-00926320

\section{HAL Id: hal-00926320 \\ https://hal-centralesupelec.archives-ouvertes.fr/hal-00926320}

Submitted on 9 Jan 2014

HAL is a multi-disciplinary open access archive for the deposit and dissemination of scientific research documents, whether they are published or not. The documents may come from teaching and research institutions in France or abroad, or from public or private research centers.
L'archive ouverte pluridisciplinaire HAL, est destinée au dépôt et à la diffusion de documents scientifiques de niveau recherche, publiés ou non, émanant des établissements d'enseignement et de recherche français ou étrangers, des laboratoires publics ou privés. 


\title{
Semi-Markov processes with semi-regenerative states for the availability analysis of chemical process plants with storage units
}

\author{
Olga Fink $^{1}$ and Enrico Zio ${ }^{2}$
}

\begin{abstract}
Design to capacity is an engineering principle that is increasingly applied in chemical industry, among others due to increasing plant sizes and associated investments. This principle aims to reduce over-capacity, oversized buffers and excessive redundancy. Concurrently, a high level of availability is targeted over the entire production chain. The consequences of unavailability of highly interconnected chemical process plants can be significant because a technical disruption in one plant is able to spread over the entire production network. In chemical process plants not only technical equipment determines the availability but also storage units, which are able to bridge times of planned or unplanned interruptions of production. To find a balance between the principle of design to capacity and high production availability, the influence of different design parameters, such as capacity of production units, redundancy concept and the size of storage units have to be evaluated and integrated in the design process. In this paper, we present an analytical method for availability evaluation based on extending Semi-Markov processes integrating storage units and multiple production lines. Semi-regenerative states are used to capture the characteristics of storage units, and an approach is proposed in this work to assign distributions for the remaining holding times in these states. The proposed modelling and analysis are demonstrated on two case studies.
\end{abstract}

\section{Keywords}

Availability analysis, chemical process plants, storage units, Semi-Markov processes, semi-regenerative states

\section{Introduction}

Market growth, steady technological advancements and the push for more profitability have amplified the principle of economies of scale in chemical industry (which also leads to increasing plant sizes), especially for basic chemical products. From the complexity side, chemical products are highly interrelated and therefore production plants are often characterized by a high degree of interconnection. This can result in complex production chains consisting of several interconnected, partly multi-channelled production plants and storage units. ${ }^{1}$ As a consequence, failures can spread over the entire production network causing, besides the direct costs of repair, also shutdown events in interconnected plants.

The availability of chemical production plants is not only determined by the availability of the technical equipment but also by that of storage units. These are designed to bridge planned or unplanned interruptions of production of single plants in a production chain.

Due to the significant impacts of the unavailability of a single plant on the connected production plants, but also due to fluctuations and uncertainties in markets, the production plants may often have over-capacities, the storage units may be oversized and the production chain may exhibit excessive redundancy. To cope with this issue, several value improving practices (VIP) are pursued in process industry, one of which is design to capacity. ${ }^{2}$ It implies a systematic evaluation of the operating capacity of the entire production chain and the individual contributions and influences of each major item of equipment. Design to capacity in the context of

\footnotetext{
${ }^{1}$ Institute for Transportation Planning and Systems, ETH Zurich, Switzerland

${ }^{2}$ Chair on Systems Science and the Energetic Challenge, European Foundation for New Energy-Electricité de France (EDF) at École Centrale Paris and SUPELEC, France;

Department of Energy, Politecnico di Milano, Italy
}

\section{Corresponding author:}

Olga Fink, Institute for Transportation Planning and Systems, ETH Zurich, Wolfgang-Pauli-Str. 15, 8093

Zürich, Switzerland.

Email: ofink@ethz.ch 
chemical process engineering aims to reduce over-capacities, oversized buffers and excessive redundancy, and thus investment costs. The application of the principle of design to capacity implies an availability evaluation of the production chain, based on plant capacities, production rates, and failure behaviour of technical equipment and storage tank sizes. Availability analysis provides a basis for specifying the optimal capacities for plants and sizes for storage units. ${ }^{3}$ As production availability is determined by both the technical equipment as well as by storage units, both must be included in the availability analysis.

There are several approaches to evaluate the availability of a process plant. Many rely on Monte Carlo simulations, ${ }^{4,5}$ for the flexibility offered to model different types of distributions, aging behaviours and maintenance policies. Some of the simulation models also include storage units in the availability evaluation. ${ }^{6,7}$ Besides simulation approaches, there are also analytical methods to evaluate the availability, ${ }^{8-10}$ but they are not flexible enough to evaluate all the relevant parameters.

The motivation of the present work is to develop an analytical method for availability evaluation of chemical production chains capable of:

- integrating storage units in the overall availability analysis;

- evaluating the availability of the total production system and of different output products, which are not necessarily the output of the last production unit in the production chain;

- evaluating the sensitivity of the capacity of a storage unit on product availability in an interconnected production chain;

- evaluating several parameters, such as the frequency of entering a certain state or the holding time spent in a defined state, which is not only limited to non-functional states;

- accounting for the possibility of operating at levels below the designed capacity.

An analytical method that can in principle integrate the stated requirements is that of Semi-Markov processes. ${ }^{11}$ However, inclusion of the states representing the storage units results in semi-regenerative states which cannot be represented by the process. An attempt to include the semi-regenerative states in the process would result in a loss of memory on the repair actions undertaken on the failed unit in cases when the storage unit is either drained or filled. To overcome this problem, an approach to assign a distribution for the remaining holding times in the semi-regenerative states is proposed in this paper. The approach is first tested on a simple case study consisting of two identical production units separated by a storage unit. The sensitivity of the volume of the storage unit on the availability of the output product is analysed. Subsequently, the approach is applied to a case study of a $C_{x}$ production chain.

In the next Section of this paper, the issues related with the integration of storage units are introduced and an overview on Semi-Markov processes is given followed by an overview over the different possibilities of integrating semi-regenerative states in the Semi-Markov processes. Then, Sections 3 and 4 illustrate the case studies and Section 5 is left for discussion and conclusions.

\section{Methodology}

\section{Integrating storage units in availability evaluation}

In process engineering, storage units are applied to decouple single production subsystems and thereby to reduce their mutual influence and the degree of interconnection. Storage units are able to increase the availability of the entire production system because decoupling of units can prevent that production units have to be shut down due to failures in the upstream or downstream of the production chain. One prerequisite of the influence of storage units on system availability is that it has to be possible to adapt the output capacity of production plants after failure events, in order to control the filling level of the storage tanks.

The ability of storage units to decouple the interdependencies of chemical production plants has a significant impact on production availability and therefore it has to be included in the availability evaluation. The impact is in two directions (Figure 1): if the upstream unit fails, the downstream unit can still be operated and the product continuously produced as long as the tank is not drained; if the downstream unit fails and its restoring duration is shorter than the buffering time of the tank, the shutting down and starting up processes of the upstream unit can be obviated although the unavailability of output production cannot be prevented. 


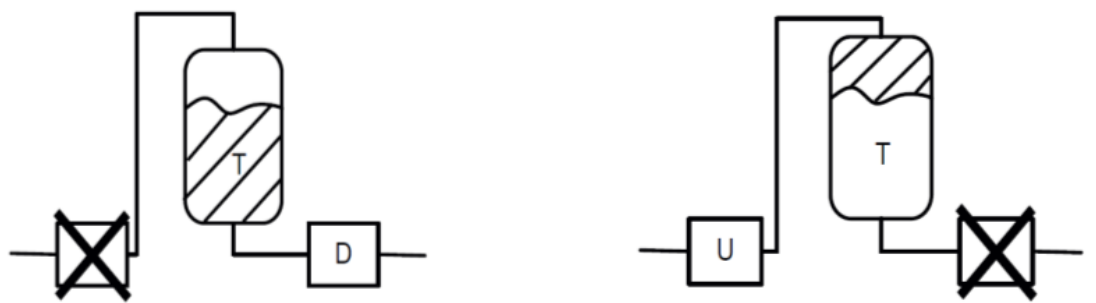

Figure 1. Bridging effect of the tank in case of failure of the upstream unit (left) or downstream unit (right).

Because the storage units have different effects on downstream and upstream units, its buffering function can be divided according to the effect: either the effect to supply the input product to the downstream unit or the effect to provide an empty storage volume for the upstream unit. In the state space, these conditions will be represented by separate states.

\section{Few concepts of Semi-Markov processes}

Semi-Markov processes belong to the class of stochastic point processes ${ }^{12}$ and are, analogously to Markov processes, regenerative processes. In the literature Semi Markov processes are also referred to as Markovrenewal processes. ${ }^{13,14}$ Equivalently to Markov processes, Semi-Markov processes can be divided into discrete and continuous processes. However, contrary to the Markov processes, which are regenerative in each single moment, Semi-Markov processes only lose their memory in state transition. The holding time in a state does not only depend on the state in which the process is in the considered moment, but also from the state which the system will change to, at the next step. ${ }^{12}$ The holding time of the state is stored and influences the state which the process makes a transition to, at the next step. The state transition is then conditional and the process only loses its memory in state transitions.

The holding time in state $i$ before the transition to state $j$ is a positive random variable with distribution function $F_{i j}(t)$. Semi-Markov processes are determined completely by defining the distribution functions $F_{i j}(t)$ of all states and specifying the initial distribution. For reliability and availability analyses, all relevant system parameters, as for example the reliability or availability of the system, can be derived from these functions. The processes are often defined by their transition probabilities $\mathrm{Q}_{\mathrm{ij}}(\mathrm{t})$ :

$$
Q_{i j}(t)=\int_{0}^{t} \prod_{\substack{k \in S \\ k \neq j}}\left(1-F_{i k}\right) d F_{i j}(x), i, j \in S
$$

with $\mathrm{Q}_{\mathrm{ij}}(\mathrm{t})$ : probability of state transition in one step from the state $\mathrm{i}$ to the state $\mathrm{j}$, within time $\mathrm{t}$ (without entering other states)

S: set of all states

$\mathrm{F}_{\mathrm{ij}}(\mathrm{t})$ : distribution function of conditional holding time $\mathrm{T}_{\mathrm{ij}}$.

The conditional probability $\mathrm{P}_{\mathrm{ij}}(\mathrm{t})$ that the system is in state $\mathrm{j}$ at time $\mathrm{t}$ if the system started at state $\mathrm{i}$ at time $\mathrm{t}=0$ can then be calculated by convolution of two probabilities:

$$
\begin{aligned}
& \left.P_{i j}(t)=\delta_{i j}\left(1-\sum_{k \in S} Q_{i k}(t)\right)+\sum_{k \in S} d Q_{i k}(t) * P_{k j}(t)\right), i, j \in S \\
& \text { with } \delta_{i j}= \begin{cases}=0, \text { for } & j \neq i \\
=1, \text { for } & j=i\end{cases}
\end{aligned}
$$

with S: set of all states

$\delta_{\mathrm{ijj}}$ : Kronecker's delta

With this equation it is possible to calculate also the reliability of the system $\mathrm{R}_{\mathrm{i}}(\mathrm{t})$. In this case, only the conditional probability of not-entering the down-states, but only remaining in the up-states, is calculated: 


$$
R_{i}(t)=\left(1-\sum_{j \in S} Q_{i j}(t)\right)+\sum_{j \in U S} d Q_{i j}(t) * R_{j}(t), i \in S
$$

with

S: set of all states

US: set of all up-states

\section{Integrating semi-regenerative states in Semi-Markov processes}

One of the advantages of applying Semi-Markov processes is that it is possible to use different types of holding time distributions, whereas Markov processes are limited to exponential distributions. However, Semi-Markov processes have limitations due to the fact that they are regenerative in each state transition. Therefore, they can only be applied for systems which can be modelled by regenerative processes. In order to expand applicability of Semi-Markov processes, non-regenerative states have to be integrated. This implies that between two renewal points state changes are allowed which are not regenerative. ${ }^{13}$ In some literature sources, a new class of semi-regenerative processes for this extension has been introduced. ${ }^{15}$ In some references, this enhancement is referred to as Markov-Renewal processes with some non-regenerative states. ${ }^{16}$ There are different approaches to integrate the evaluation of non-regenerative states, but not all of them fulfil the requirements of a new process class. Therefore, the enhancement induced by integrating nonregenerative states is referred to as an extension of Semi-Markov processes and not as a new class of processes. In the following, an overview of different approaches to integrate semi-regenerative states in the evaluation of Semi-Markov processes is introduced.

One of the possibilities for integrating semi-regenerative states is based on the condition that the process changes from each semi-regenerative state to a regenerative state ${ }^{15}$ and that only one regenerative state can be entered from a semi-regenerative state.

Nakagawa and Osaki introduced in ${ }^{16}$ another possibility to evaluate semi-regenerative states. This approach introduces a new class of transition probabilities and the process either transfers through several cyclic semiregenerative states before it re-enters a regenerative state or the process always re-enters a regenerative state before it can enter a semi-regenerative state.

Another possibility to evaluate semi-regenerative states is to identify the distribution of the remaining holding times. If the distributions of the remaining holding times can be defined, semi-regenerative states merge into regenerative states and the evaluation of the process can be based on Semi-Markov processes. However, in practice it is usually difficult to determine these distributions. Therefore, typically, assumptions are made on the distribution of remaining holding times.

A further possibility to evaluate Semi-Markov processes with semi-regenerative states is to transfer the whole state space into a Markov process. At first glance this approach seems not to have any advantage over setting up directly a Markov process, without having to detour to Semi-Markov processes. But the holding times in single states cannot always be assumed or approximated to be exponentially distributed. The direct assumption of constant transition rates would lead to a loss of information and inaccuracies in computation results. Approximations of Semi-Markov processes by Markov processes intend to integrate memory in the approximated processes and by this to reduce loss of information and inaccuracies, but at the same time to facilitate the computation of the modelled processes. Therefore, this approach is more powerful compared to pure Markov processes.

There are numerous ways to approximate Semi-Markov processes by Markov processes. One of the possibilities is to introduce supplementary variables that transfer a non-Markovian process in a Markov process (Non-Markovian processes are stochastic point processes, which do not have the Markovian attributes and include Semi-Markov processes but are not limited to them ${ }^{17}$ ). The introduced supplementary variables are predominantly time variables. They represent holding times in semi-regenerative states, as for example the remaining duration of a repair activity. The state space is thereby transferred in a multidimensional state space. ${ }^{18,19}$ This method can always be applied if parts of the process already incorporate the Markovian assumption. ${ }^{20}$ The application of the method is limited to systems with only few components and a limited number of supplementary variables. ${ }^{21,}{ }^{22}$ The reason for this limitation is that 
typically, not only one supplementary variable is required but several, and the state space can be significantly extended by this approach.

A further possibility to approximate Semi-Markov processes by Markov processes is limited to Erlang distributed holding times and involves the introduction of auxiliary transition steps. ${ }^{14}$ An Erlang distribution represents systems that consist of several components with identical failure behaviour. It is formed by an $\mathrm{n}$ fold convolution of the exponential distribution with itself, which corresponds to the sum of $n$ identical exponentially distributed random variables. Due to this property of the Erlang distribution, the Semi-Markov process described by Erlang distributed holding times can be directly transferred in an equivalent Markov process. The number of auxiliary steps and the transition rates between the single states are indicated by the distribution function. This approach leads to an expanded state space due the introduction of auxiliary transition states.

A generalization of the approach of auxiliary steps has been introduced in ${ }^{23}$ with the phase-type distribution, which is also referred to as ph-distribution. Phase-type distributions are characterized by a finite, absorbing Markov process. A phase-type-distributed variable of $n$-th order represents the time up to absorption in a Markov process with $n$ states. Generally, there is a distinction between discrete and continuous phase-type distributions, but only the latter is relevant for approximatimg Semi-Markov processes. By applying phasetype distributions, it is not the processes that are approximated but the distributions of the holding times. Some distributions are particularly suitable for approximations by the phase-type distributions, such as hyper-exponential distribution or its generalization in the Cox distribution. ${ }^{24}$ The distributions can be substituted by several combined exponential distributions: thereby, transition rates are constant. The approximation process is a two-step process. First, a suitable distribution or combination of distributions for approximation is selected. Thereafter, the number of steps and the parameters of the distribution are determined. This approach provides a good approximation for arbitrary distributed functions when applied with an appropriate number of steps. The memory of processes is created indirectly by the number of steps in the distribution.

Another possibility to approximate Semi-Markov processes by Markov processes is to determine constant equivalent transition rates between the states. The prerequisite is that relevant information in the model is not lost. A central assumption for approximating Semi-Markov processes by Markov processes is that the considered processes are ergodic, which means that they are not dependent on initial conditions. Time dependent Semi-Markov processes are non-ergodic. Limnios states in ${ }^{11}$ that for finite time periods the determination of state probabilities with approximated processes is inaccurate. But stationary processes can be approximated by equivalent transition rates. For obtaining the equivalent transition rates the following equations have to be solved:

$$
\lambda_{i j}^{\prime}=\frac{p_{i j}}{T_{i}}, i, j \in S
$$

with $\quad \lambda_{\mathrm{ij}}$ : equivalent transition rate from state $\mathrm{i}$ to state $\mathrm{j}$

$\mathrm{p}_{\mathrm{ij}}$ : probability of one step transition from state $\mathrm{i}$ to state $\mathrm{j}$

$\mathrm{T}_{\mathrm{i}}$ : holding time of the process in state $\mathrm{i}$, before the process changes to an arbitrary state

with

$$
p_{i j}=\lim _{t \rightarrow \infty} Q_{i j}(t), i, j \in S
$$

and

$$
T_{i}=\int_{0}^{\infty}\left(1-H_{i}(t)\right) d t=\int_{0}^{\infty}\left(1-\sum_{j \in S} Q_{i j}(t)\right) d t, i, j \in S
$$

with $\quad \mathrm{H}_{\mathrm{i}}(\mathrm{t})$ : distribution function on non-conditional holding time $\mathrm{T}_{\mathrm{i}}$ 
For the purposes of the work presented in this paper, we have adopted the method of determining remaining holding times for semi-regenerative states combined with the method of equivalent Semi-Markov transition rates. The latter is applicable to not time-dependent Semi-Markov processes, but the inaccuracies which can occur in a finite time space are accepted in our case. Additionally, in order to deduce the state space of the obtained Markov processes, similar states are aggregated by determining equivalent transition rates.

\section{Verification of the concept on a simple case study}

A simple system was selected in order to verify the proposed modelling approach. The system consists of two units which are connected by a storage unit (Figure 2). The units are assumed to have the same failure behaviour with equal constant failure and repair rates. Then, the time between failures and the time to restore the function are assumed to be exponentially distributed. This assumption was only made for simplicity reasons and can be easily relaxed. The mean time between failures (MTBF) is equal to 800 operating hours and the mean time to restore the units and put them back to operation (MTTR) is equal to 25 hours. Additionally, the units have the same production rate, which is equal to $20 \mathrm{~m}^{3} / \mathrm{h}$, so that the tank can be filled and drained with the same rate. The decoupling time of the tank can be computed based on the capacity of the tank and the filling and the draining rate, respectively. This is the time during which the tank can be considered as a redundant system that supplies the product. During this time, the units are decoupled and the failures of one unit do not influence the production of the other unit. The tank can only fulfil this function for a limited period of time, the decoupling time $\left(\mathrm{T}_{\mathrm{D}}\right)$.

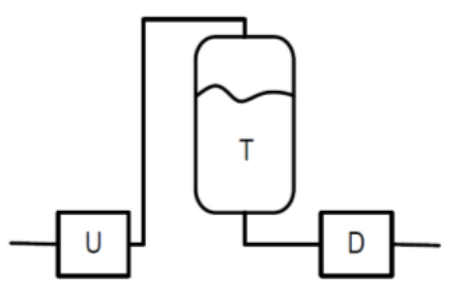

Figure 2. Simple system with an upstream and a downstream unit decoupled by a storage tank.

It is additionally assumed that the production capacity of the units can, for a short period of time, exceed the nominal value. Thereby, after a failure event the units are able to produce with a higher production rate in order to be able to regulate the filling level of the tank. If it were not possible to increase the production rate above the nominal, the time to put the production system back into full operation would increase or the output production would decrease until the desired filling level of the tank is achieved.

Figure 3 demonstrates the state space diagram of the considered system, consisting of six states (which are numbered and coded). State 1 is the only state that is fully operable. In state 3 the output product can be produced in full amount. In state 2, even though the output product cannot be produced, the upstream unit can still be operated and does not have to be shut down. For simplicity reasons, it is assumed that if both units fail, they are taken together back into operation, which corresponds to state 4 . This assumption makes perfectly sense in terms of practical considerations. States 5 and 6 are semi-regenerative states. Making them regenerative in first place would mean that the repair works that have already been performed on the failed units would have to be restarted when the tank is drained or filled. This would lead to significant inaccuracies in calculations. Therefore, distributions for the remaining holding times are assigned to these states in order to make them regenerative while preserving accuracy in calculations. 


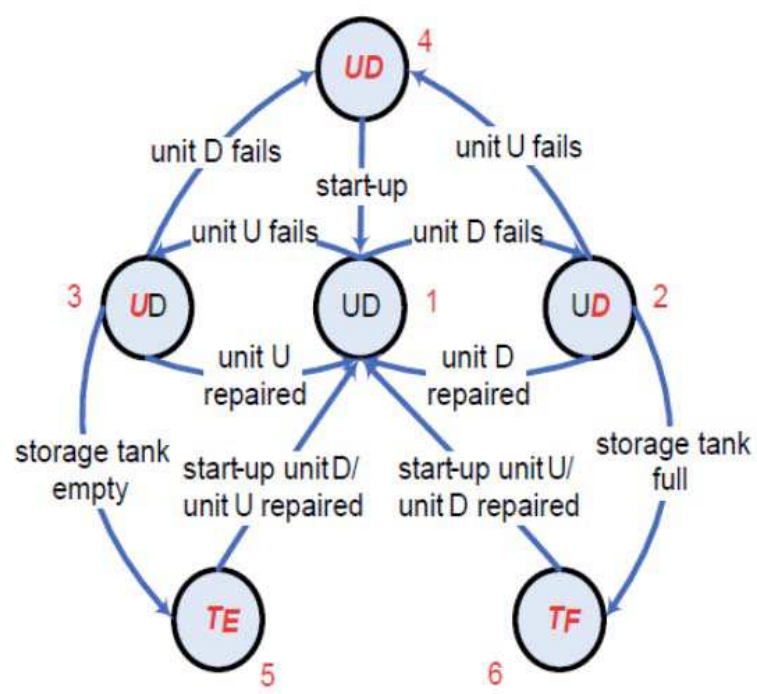

Figure 3. State space diagram of the considered system.

U: upstream unit; $D$ : downstream unit, $\mathrm{T}_{\mathrm{E}}$ : storage unit empty; $\mathrm{T}_{\mathrm{F}}$ : storage unit filled; states marked red: production unit failed or storage unit not fulfilling its function .

The filling level of the storage tank is assumed to be regulated and therefore can be considered as deterministic. It is regulated to a filling level of $50 \%$ of the total tank capacity. Thereby, down- and upstream units are regarded as being equally important. Based on this assumption we can deduce the distribution for the remaining holding times in the semi-regenerative states. Therefore, it is assumed that the tank can buffer the portion of the restoring times that are smaller than the decoupling time of the storage tank. The part of the density function for which $t>T_{D}$ is assigned to the remaining holding time of the semi-regenerative states. The density function is divided therefore in two parts. For the time smaller than the expected decoupling time of the tank, the following density function is assigned: $\mathrm{P}\left(\mathrm{t}>\mathrm{T}_{\mathrm{D}}\right)=\mu \mathrm{e}_{\mathrm{D}}^{-\mu(\mathrm{t}-\mathrm{T})}$. In Figure 4 the assigned density function to the semi-regenerative states is marked hatched.

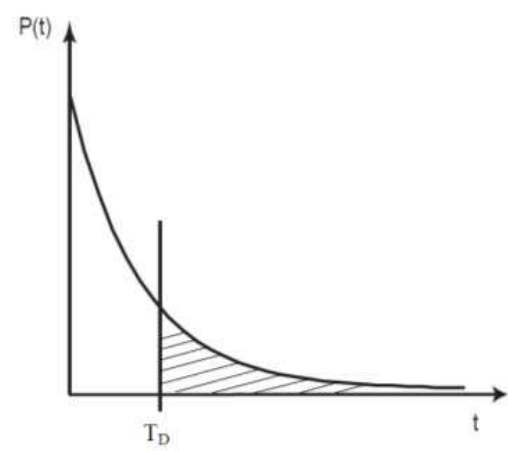

Figure 4. Distribution of the remaining holding times in the semi-regenerative states.

By assigning the distribution to the remaining holding times for semi-regenerative states, all the conditional and unconditional transition probabilities can be calculated. The sensitivity of the decoupling effect of the tank volume is analysed. Assuming that in the first place we are only interested in the availability of the production output, we will consider the influence of the tank volume on the mean time between successive production unavailabilities. There are two extremes: when the units are operated in series and are not decoupled, and when the tank has a sufficient volume to completely decouple both of the units so that only the downstream unit determines the number of shutdowns and therefore also the number of times when the production is not available. Figure 5 shows the relationship between the capacity of the tank unit and the mean time between successive production unavailabilities which arise at shutdown of the downstream unit, due either to failure of the unit itself or if the tank volume is not able to supply the input product in case of a failure of the upstream unit. With a tank volume of approximately $5000 \mathrm{~m}^{3}$ both units are decoupled and only the failures of the downstream unit determine the unavailability of the production output. 


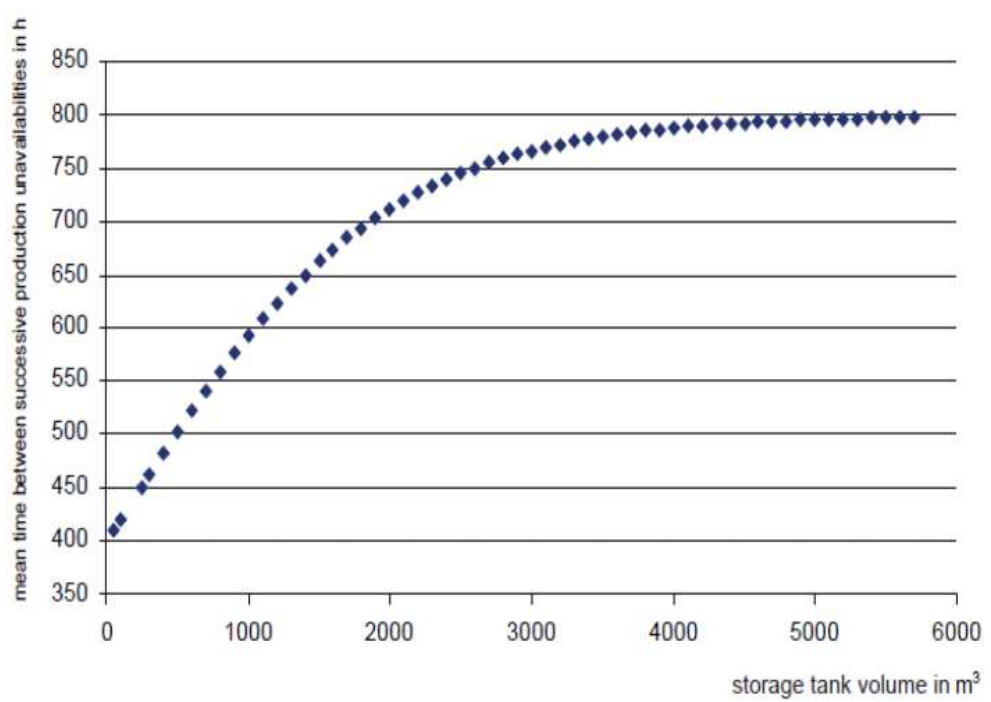

Figure 5. Impact of storage tank volume on the mean time between successive production unavailabilities.

\section{Application to a $\mathrm{C}_{\mathrm{x}}$ production chain}

A $C_{x}$ production chain is considered ( $x$ substitutes a number in organic carbon compounds, due to information protection). The block diagram of the production chain is shown in Figure 6. Real failure and repair data were alienated due to information protection, but without a loss of significant information and characteristics.

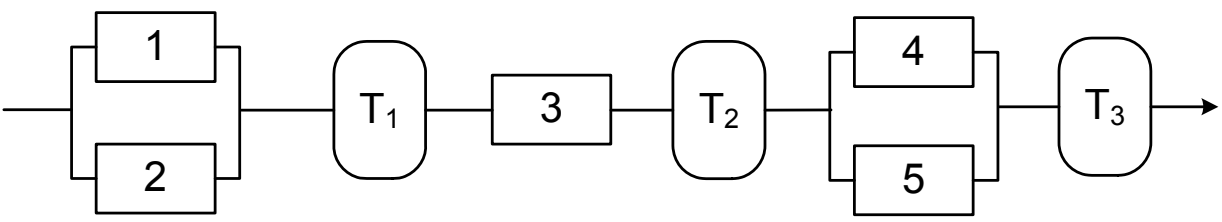

Figure 6. Block diagram of $\mathrm{C}_{\mathrm{x}}$ production chain.

The chemical production system consists of five plants (units 1-5) and three storage units $\left(T_{1}-T_{3}\right)$. The plants that are shown in Figure 6 as being redundant, i.e. units 1 and 2, and units 4 and 5, are multi-channelled and not fully redundant. The capacities of the single multi-channelled units add up to $100 \%$ of the required capacity in the next production step. If one of the multi-channelled units fails, the upstream and downstream units are usually still able to operate, but with a reduced production capacity. The capacity proportions of the single units of the total production capacity in each production step are listed in Table 1. Additionally, the failure and restore behaviour of the single units is specified. For failure behaviour, only information on the frequency of random failures was available. As the exact distribution could not be determined due to lack of data, exponential failure behaviour was assumed.

Table 1. Failure and repair behaviour of units in the production chain.

\begin{tabular}{llll}
\hline Production unit & \% of total capacity & MTBF in h & MTTR in h \\
\hline 1 & 33.33 & 14,600 & 72 \\
2 & 66.67 & 14,600 & 72 \\
3 & 100 & 4380 & 72 \\
4 & 75 & 8760 & 24 \\
5 & 25 & 876 & 48 \\
\hline
\end{tabular}

The maximum volumes of the storage units are listed in Table 2.

Table 2. Maximum volumes of storage units.

\begin{tabular}{ll}
\hline Storage unit & Volume of the storage unit in $\mathbf{~ m}^{\mathbf{3}}$ \\
\hline 1 & 2000 \\
2 & 2800 \\
3 & 2000 \\
\hline
\end{tabular}


Availability considerations not only integrate the influence of random events, but also include the influence of planned maintenance activities. Planned preventive maintenance activities have a deterministic character. The present analysis only focuses on time-based maintenance scheduling. Generally, it is possible to coordinate into groups single maintenance activities of the production units. This approach is not included in this study, and therefore the maintenance activities are considered separately. The frequency and duration of the individual maintenance activities are listed in Table 3.

Table 3. Frequency and duration of planned maintenance activities.

\begin{tabular}{lll}
\hline Production unit & $\begin{array}{l}\text { Frequency of planned } \\
\text { maintenance activities }\end{array}$ & $\begin{array}{l}\text { Duration of maintenance } \\
\text { activities in d }\end{array}$ \\
\hline \multirow{2}{*}{1} & twice in 5 years & 7 \\
& once in 5 years & 35 \\
\hline \multirow{2}{*}{2} & twice in 5 years & 7 \\
& once in 5 years & 35 \\
\multirow{2}{*}{3} & once in 5 years & 35 \\
& twice in 5 years & 21 \\
\hline \multirow{2}{*}{5} & once in 2 years & 5 \\
& once in 5 years & 16 \\
\hline
\end{tabular}

For the case study, the following assumptions were made to facilitate the computations. The same assumption made in the previous case study on the filling level of the storage tank in case of a failure event is adopted ( $50 \%$ of the total tank capacity). In general, this assumption can be relaxed and the filling level can be optimized depending on the relative failure behaviour of the up- and downstream units. But for this calculation, the optimization of the filling level is not in the focus of the study. In case of planned deterministic events, it is assumed, that the filling level of the tank is anticipated and the tank is either drained or filled.

Furthermore, it is assumed that in case of multi-channelled units, if both of them fail, they are started up simultaneously. If one of the multi-channelled units has already failed and the second unit fails before the tank is drained or filled, the filling level is assumed to be $75 \%$, respectively $25 \%$, of the tank capacity. If one of the multi-channelled units fails, storage tanks cover the lost capacity for the period during which the tank volume is available. Thereafter, all the connected production units reduce their production capacity to the maximal capacity of the functioning multi-channelled unit which is still in operation. It is assumed that it is possible for all the production units to reduce their product output to these production levels. Furthermore, this approach neglects that other storage units in the production chain could also buffer the capacity gap of the up- or downstream units. This assumption reduces significantly the state space of the Semi-Markov process. The inaccuracies caused by this assumption are negligible.

In the first step only the influence of random events is analysed. The deterministic analyses are performed separately and are included in the state space of Semi-Markov processes. But it is generally possible to perform the analysis by including random and deterministic events in one single Semi-Markov process.

To reduce the complexity of the resulting state space, the system is divided in three subsystems, as shown in Figure 7. The first subsystem includes the units 1 and 2 , and the storage unit $T_{1}$. The second subsystem includes the unit 3 and the storage units $T_{1}$ and $T_{2}$. The third subsystem integrates the units 4 and 5 , and the storage unit $T_{3}$. The subsystems are first analysed separately; if necessary, the number of states is reduced. If the states are not feasible from the practical point of view, they are not included in the considerations. The reason that the storage unit $T_{1}$ is included twice in the subsystems is that storage units have always an influence on the upstream units and on the downstream units. For storage unit $\mathrm{T}_{2}$, it is covered by the combination of the subsystems. 


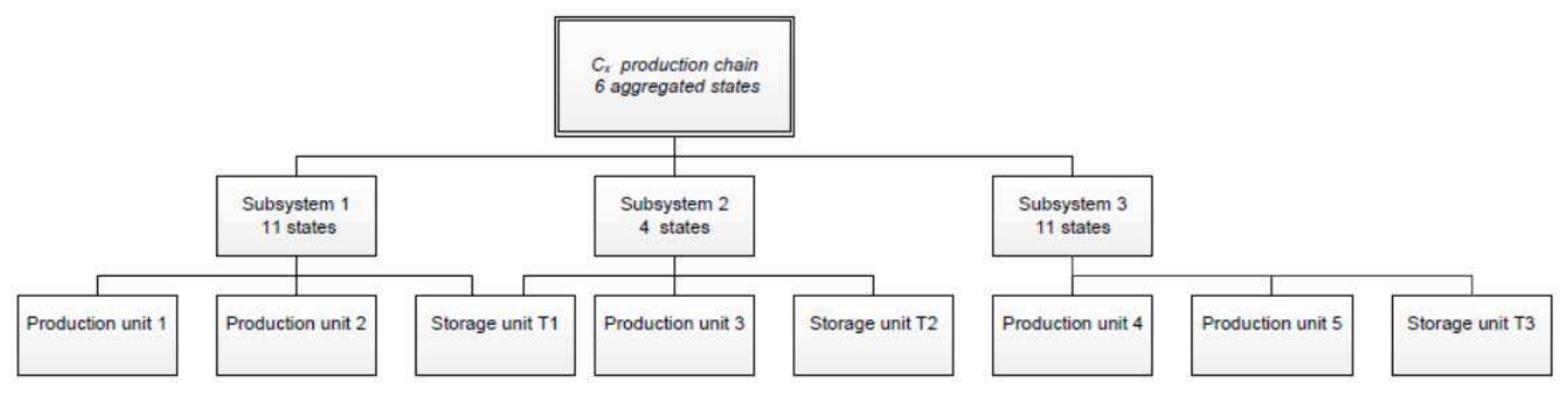

Figure 7. Division of the $\mathrm{C}_{\mathrm{x}}$ production chain into subsystems for availability analysis.

The models of subsystems 1 and 3 result in 11 states, each; the model of subsystem 2 results in 4 states. The pertinent state spaces are shown in Figure 8. Subsequently, the transition probabilities and holding times of the subsystems are computed. Based on these data, the states of the subsystems are aggregated and the state space is reduced. When combining the subsystems, the interaction of the subsystems is considered and supplementary states are created if required. When combining the subsystems, some of the states are not feasible. These states are excluded from further consideration. The transition probabilities and the holding times of the merged subsystems are calculated and if required the state space is reduced, or the states are aggregated.

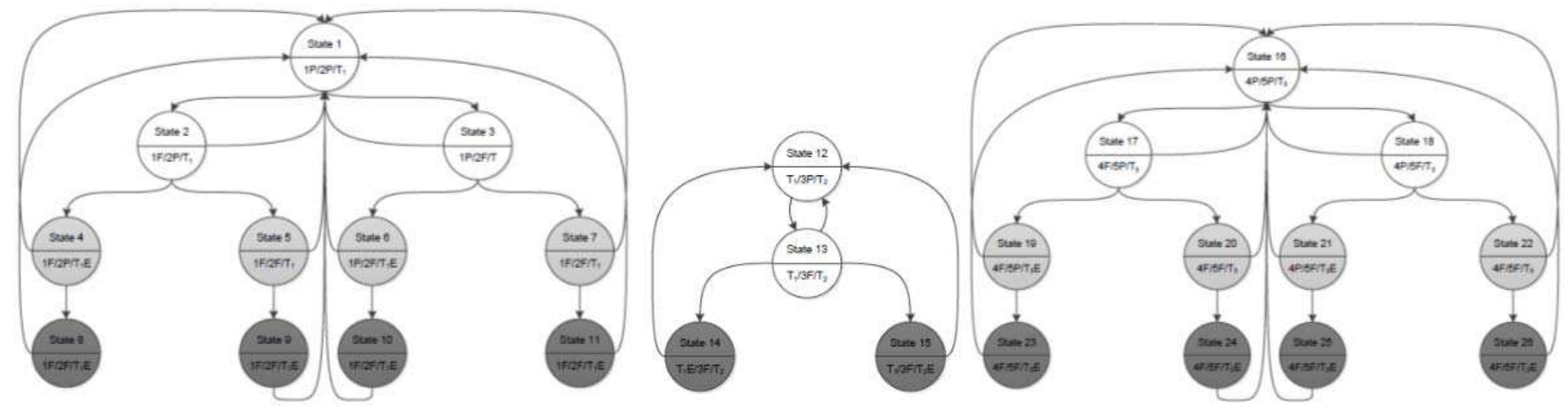

Figure 8. State spaces of the three subsystems (subsystem 1: left, subsystem 2: middle, subsystem 3: right). P: unit in up-state; F: unit failed; T: storage unit not affected; TE: storage unit empty or filled; states marked in a shade of grey: production output degraded.

In the following, the results of the availability analysis are presented. The production chain can be fully functional and have a $100 \%$ production output (state 1) or it can be in states with reduced production output (states 2-5). There are four possible states with a degraded production output $(75 \%, 67 \%, 33 \%$ and $25 \%$ of the total production capacity) and one non-functional state (state 6). The results of the analysis are shown in Table 4. The probabilities to be in the considered state and the average stationary holding times in the states are listed in the Table. The analysis shows that the product is on average unavailable only in $0.6 \%$ of the time and if the production is unavailable it takes on average 23 hours to restore it. The storage unit can buffer a large part of the repair time of single units, which can be seen in the comparison of the holding times in the degraded states that vary on average between 4 and 43 hours, and times to repair single units that vary between 24 and 72 hours. Additionally, the storage units can prevent the system from entering a state with a degraded production output in cases when the repair times are shorter than the bridging times of the storage units. State 5 with the maximal production output of $25 \%$ is the degraded state with the longest average holding time of 43 hours and the highest probability of $0.22 \%$. This state is determined by the most unreliable unit in the whole production chain (unit 5) and the repair times of this unit can only be buffered partly by the storage unit. 
Table 4. Computational results of the case study.

\begin{tabular}{llll}
\hline $\begin{array}{l}\text { Aggregated } \\
\text { state } \\
\mathbf{j}\end{array}$ & $\begin{array}{l}\text { Percentage of maximal } \\
\text { production output, } \\
\text { in \% }\end{array}$ & $\begin{array}{l}\text { Probability to be in state } \mathbf{j}, \\
\text { in \% }\end{array}$ & $\begin{array}{l}\text { Average stationary } \\
\text { holding times of the } \\
\text { system in the state, in } \\
\text { h }\end{array}$ \\
\hline 1 & 100 & 99.1180 & 3407.17 \\
2 & 75 & 0.0281 & 18.22 \\
3 & 67 & 0.0199 & 3.98 \\
4 & 33 & 0.0259 & 5.36 \\
5 & 25 & 0.2231 & 43.21 \\
6 & 0 & 0.5850 & 22.94 \\
\hline
\end{tabular}

The impacts of the planned maintenance activities are analysed separately from the stochastic events. With the assumptions described previously, the results shown in Table 5 are obtained. The percentage of the time was calculated in which the system is not able to provide the total required production capacity due to the planned maintenance activities.

Table 5. Reduced production output due to planned maintenance activities.

\begin{tabular}{lll}
\hline $\begin{array}{l}\text { Aggregated state } \\
\mathbf{j}\end{array}$ & $\begin{array}{l}\text { Percentage of maximal } \\
\text { production output, in \% }\end{array}$ & $\begin{array}{l}\text { Percentage of the time the } \\
\text { system is in state j, in \% }\end{array}$ \\
\hline 7 & 75 & 0.71 \\
8 & 67 & 0.60 \\
9 & 33 & 0.44 \\
10 & 25 & 0.37 \\
11 & 0 & 3.12 \\
\hline
\end{tabular}

As the maintenance activities are planned, the volume of the storage units can be regulated to the maximal buffer capacity. Therefore, some of the maintenance activities can be buffered entirely by the storage units. Other maintenance activities have a reduced impact on the availability of the product. In $3 \%$ of the time, the product cannot be supplied in full and in $2 \%$ of the time only a reduced volume of the product can be supplied. By regulating the level of the storage units and by coordinating the maintenance activities, it is possible to reduce the time in which the product cannot be supplied in full or in part to $5 \%$ in total. Without these measures the time with reduced production output would be threefold of this number.

\section{Conclusions}

By extending Semi-Markov processes with semi-regenerative states, it has been shown possible to integrate storage units in chemical production chains for their availability evaluation. By combining different approaches such as assigning distributions to the remaining holding times, determining the equivalent transition rates and aggregating of states based on transition rates and holding times, a reduction of the computational complexity could be achieved.

Some of the assumptions specified in this case study can be easily relaxed, such as the exponentially distributed failure and repair behaviour. Semi-Markov processes are flexible to be applied with any distribution.

As modelling of the state space was not automated, it was essential in this case study to maintain an overview of the system and its behaviour in different states, and to be able to understand the generated state space in terms of its technical interpretation. Therefore, the system was divided into subsystems and part of the analysis was performed at the subsystem level, before the sub-processes were combined and integrated. On the contrary, the aggregation of states reduces the flexibility of the analysis and the degrees of freedom. Therefore, the next step to expand the flexibility of this approach is to automate the analysis with having predefined components with predefined state spaces based on their failure behaviour, and then combining these predefined components into a production chain and its state space. By this approach, an overview and understanding of the technical function of the system is maintained and at the same time the flexibility is gained, without having to reduce the state space. 
In the application, the load profiles of the single plants were not considered. Including the load profiles in the analysis would additionally increase the state space. However, it is possible to easily integrate different load profiles with the proposed approach.

As discussed in Section 2, it is possible to approximate Semi-Markov processes by the phase-type distribution approach. The proposed approach did not follow the phase-type distribution approach, which might be beneficial to include in future research.

The paper proposes an approach to include semi-regenerative states in Semi-Markov processes by assigning distributions for the remaining holding times in these states. This approach is adopted for applying it to availability evaluation of chemical production chains with storage units. By assuming regulated filling levels of the storage units, the density function representing repair times larger than the bridging time of the storage units is assigned to the semi-regenerative states.

The proposed approach enables analytical availability evaluations which include both, the stochastic failure behaviour and the buffering influence of storage units. Additionally, the approach enables evaluations of all relevant parameters such as holding times in different states, and conditional and unconditional probabilities. Even though some assumptions may cause some inaccuracies in the results, the overall level of accuracy is appropriate for applying the approach in the design or modification phase, to enable an optimal layout of production network and to support decisions.

\section{References}

1. Koolen JLA. Design of Simple and Robust Process Plants. Weinheim: Wiley-VCH Verlag GmbH \& Co. KGaA, 2003.

2. Lees' Loss Prevention in the Process Industries (Fourth Edition). Oxford: Butterworth-Heinemann, 2012, p.443-508.

3. Koolen JLA, Sinke DJ and Dauwe R. Optimization of a total plant design. Computers \& Chemical Engineering. 1999; 23, Supplement: S31-S4.

4. Dubi A. Analytic \& Monte Carlo methods for realistic systems analysis. Mathematics and Computers in Simulation. 1998; 47: 243-69.

5. Borgonovo E, Marseguerra $\mathrm{M}$ and Zio E. A Monte Carlo methodological approach to plant availability modeling with maintenance, aging and obsolescence. Reliability Engineering \& System Safety. 2000; 67: 6173.

6. Fabricius SMO. Modeling and simulation for plant performability assessment with application to maintenance in the process industry. Zürich2003, p.202 S.

7. Hamada M, Martz HF, Berg EC and Koehler AJ. Optimizing the product-based availability of a buffered industrial process. Reliability Engineering and System Safety. 2006: 1039-48.

8. Wijngaard J. The Effect of Interstage Buffer Storage on the Output of Two Unreliable Production Units in Series, with Different Production Rates. AIIE Transactions. 1979; 11: 42-7.

9. Malathronas JP, Perkins JD and Smith RL. The Availability of a System of Two Unreliable Machines Connected by an Intermediate Storage Tank. IIE Transactions. 1983; 15: 195-201.

10. Henley EJ and Hoshino H. Effect of Storage Tanks on Plant Availability. Industrial \& Engineering Chemistry Fundamentals. 1977; 16: 439-43.

11. Limnios N and Oprisan T. Semi-Markov processes and reliability. Boston: Birkhäuser, 2001, p.222 S.

12. Howard RA. Semi-Markov and decision processes. New York [etc.]: Wiley, 1971, p.XVIII, S. $577-$ 1108, I-1-12.

13. Gaede K-W. Zuverlässigkeit - mathematische Modelle. München Wien: Hanser, 1977, p.249 S.

14. Ravichandran N. Stochastic methods in reliability theory. New York [etc.]: Wiley, 1990, p.VIII, 201 S.

15. Bernet RE. Modellierung reparierbarer Systeme durch Markoff- und Semi-regenerative Prozesse. Zürich1992, p.IV, $143 \mathrm{~S}$.

16. Osaki S and T. N. Markov renewal processes with some non-regeneration points and their application to reliability theory. Microelectronics and Reliability. 1976: 633-6.

17. Srinivasan SK. On a class of non-Markovian processes. Madras1962, p.15 Bl.

18. Cox DR and Miller HD. The theory of stochastic processes. [Repr.] ed. London: Chapman and Hall, 1984, p.398 S.

19. Alfa AS and Rao TSSS. Supplementary variable technique in stochastic models. Probability in the Engineering and Informational Sciences. 2000; 14: 203-18. 
20. Singh C and Billinton R. System reliability modelling and evaluation. London: Hutchinson, 1977, p.VII, 248.

21. Whitt W. Continuity of generalized semi-markov processes. Mathematics of operations research. 1980; 5: 494-501.

22. Haddad S and Moreaux P. Approximate analysis of non markovian stochastic systems with multiple time scale delays. The IEEE Computer Society's 12th Annual International Symposium on Modeling, Analysis, and Simulation of Computer and Telecommunications Systems (MASCOTS). Volendam2004, p. 2330 .

23. Neuts MF. Matrix-geometric solutions in stochastic models an algorithmic approach. New York: Dover, 1994, p.XIII, 332.

24. Cox DR, Hand DJ and Herzberg AM. Selected Statistical Papers of Sir David Cox. Cambridge: Cambridge University Press, 2006. 